\title{
Paediatric Focused Triggering Tool (PFTT) To Assess the Harm and its Utilization to Minimize the Levels of Harm among Children at a Tertiary Care Hospital
}

\author{
Geetanjali Shankarprasad Salimath*, Madiwalayya Shivakantayya Ganachari, Manjula Gudhoor \\ Department of Pharmacy Practice, KLE College of Pharmacy, KLE Academy for Higher Education and Research (KAHER), Belagavi, \\ Karnataka, INDIA.
}

\begin{abstract}
Objectives: Very few paediatric trigger tools have been developed to assess harm in children. Hence, a Paediatric-focused trigger tool has been developed, assess its utility to measure the levels of harm, categorize and minimize them further. Methodology: The final 40 trigger tool developed by modifying IHI-GTT adult care, was prospectively tested for structural review of records $(n=520)$ of paediatric population of a tertiary care hospital for incidence of adverse events ( $A E^{\prime} s$ ) with associated harm and categorized using modified NCCMERP (National Coordinating Council for Medication Error Reporting and Prevention) index from E-I. Results: Among 292 male and 228 female children, the incidence of AEs was $35 \%$, more in the category of 1-5 years of age with harm assessment events. Out of 182 AEs identified, $107(58.80 \%)$ were found to be of E-category, $43(23.62 \%)$ were of category F, 22(12.08\%) with category G, 9(4.95\%) with category $\mathrm{H}$ and $1(0.55 \%)$ was category I. Two third of the paediatric population of category $E$ and $F(82.4 \%)$ had temporary harm and remaining $(17.6 \%)$ were of nonpreventable harm, category G-I. One example of AE identified with harm was, decrease in $25 \%$ in Haemoglobin or Haematocrit and number of blood transfusions. Further action was taken to strictly monitor and minimize further harm. Conclusion: The present study highlights on the harm assessment among the paediatric population with the help of developed Paediatric-Focused Trigger Tool (PFTT) modified by using IHI-GTT adult trigger tool. This helps paediatric set ups to assess its usefulness and further develop strategies to mitigate the harm.
\end{abstract}

Key words: Paediatric, Trigger tool, AEs, NCCMERP Index, Harm.

\section{INTRODUCTION}

To achieve better patient care among children, efforts are being made to minimize the substantiated harm in the children which may be iatrogenic in nature. The efforts towards the same can allow for improvement in paediatric patient care and institutional healthcare policy. One such effort from the 'Institute for Healthcare Improvement's Global Trigger Tool (IHIGTT), has become standard practice to identify and measure rates of AEs. ${ }^{1-3}$

Patient harm over the period and its assessment in an accurate way remains as a challenge for most of the organizations. Only $2 \%$ to $8 \%$ of all harms has been detected through voluntary reporting, which is the common method of detection of harm detection in most of the hospitals. Different studies showed that prevalence of adverse events (AEs) in hospitalised adult population range from $2.9 \%$ to $16.6 \%{ }^{4,5}$ Hence, concurrent use of complementary methods are used instead of the single voluntary method and has proved to be beneficial for the detection of adverse events. ${ }^{1,6-11}$ One such method known as 'Trigger tool (T'T)' methodology, is considered to be the best single tool at present time. Also, it is one of the reliable and sensitive tools for detecting the adverse event related
Submission Date: 25-01-2020; Revision Date: 19-04-2020; Accepted Date: 17-06-2020

DOI: 10.5530/ijper.54.3.134 Correspondence: Mrs. Geetanjali S Salimath Assistant Professor, Department of Pharmacy Practice, KLE College of Pharmacy, KLE Academy for Higher Education and Research (KAHER), Belagavi-590010, Karnataka, INDIA.

Phone: +919902687176

E-mail: geetanjalisalimath@ gmail.com

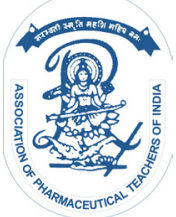

www.ijper.org 
harm more accurately over the time by customising to different settings. The landmark Harvard Medical Practice Study has used screening method for reviewing charts to detect harm efficiently. ${ }^{12}$

The idea of a 'trigger' (clue) to detect adverse events in the patient's medical records was introduced by Jick in the year 1974, 13,14 and manual record review using the triggers was initially developed by IHI in 1999 to identify adverse medication events. A 'trigger' is "an existence of, or signal in the patient medical records that may indicate an $\mathrm{AE}$ and serve as a clue or a hint indicating reviewers towards thorough investigation of the records, they focus on patient harm, not errors'. ${ }^{15,16}$ Once the harm is identified, causes and contributing factors should be assessed, then solutions to be found to increase the safety of care (WHO 2014b). ${ }^{17}$

The present study, using the developed paediatricfocused trigger tool (PFTT) at the paediatric setting, is a potential approach to measure the level of harm, assigning category and mitigate them in the paediatric population of a tertiary care teaching hospital.

\section{MATERIALS AND METHODS}

\section{Setting}

There was a need for determining the utility of IHI-GTT adult care in paediatric set up of different age groups at the institutions level of a large tertiary care hospital. After obtaining institutional Ethics Committee approval, a paediatric focused triggering tool (PFTT) was applied in different settings of the paediatric population to, delete triggers not positively predicting the adverse events and add more relevant triggers. Primary focus of this study was to examine the extent of harm through developed PFTT and secondly, to assess the usefulness of the different triggers, which includes the utility of individual triggers in identifying the harm.

Five trigger tools were identified from different literature search engines for IHI-GTT adult care tool, some triggers were added and modified with duplicate triggers eliminated. Adopting the modified Delphi process, the tool with 61 triggers was modified to a final tool with 40 triggers. It was prospectively tested for a structural review of records (SRR). To achieve validation, 20 charts every month were randomly selected across five age groups. 520 charts were reviewed by two internal primary reviewers (Clinical Pharmacists) with knowledge of and trained for identifying AE's and one external reviewer (Clinician/Physician) with knowledge of and expatriation in the similar field. The prevalence of triggers and positive charts with AEs were recorded, charts with AEs identified by Pharmacists and validated physicians were determined for positive predictive value.

\section{Patient chart review of random Samples}

Developed final trigger tool (40 final trigger list selected from the retrospective chart review process, carried out in stage I process of the study by modifying an adult version of IHI GTT) was used to assess the charts or records. The developed PFTT contains five groupings of modules or triggers. Five of the modules are developed to indicate for prevalence of adverse events that commonly appear in a paediatric patients; the five modules are:

- Care

- Medication

- Surgical

- Emergency and

- Laboratory

\section{The Sampling process of the Patient Records/} charts

A prospective random sample of 520 records (by computerized randomization method) were selected to identify adverse events Assessed for harm from the patients of paediatric department of a tertiary care hospital each month. Every $10^{\text {th }}$ admission/discharge record was selected for reliability.

\section{Events Review and assessment by the Reviewers}

- Harm assessment

- Severity Reviewed

\section{The review team consisted of 03 people}

1) Two primary record reviewers with pharmacotherapeutic background and knowledge about the contents of the assessment

2) A physician who authenticates or further validates the agreement of the two primary record reviewers.

\section{Assigning category of 'harm'}

As per the "National Coordinating Council for Medication Error Reporting and Prevention (NCC MERP)", harm was categorised and actions were taken to minimize it.

\section{Definition of Harm}

"Temporary or permanent impairment of the physical, emotional, or psychological function or structure of the body and/or pain requiring intervention" as per the NCC MERP definition. ${ }^{18}$ Accordingly, the PFTT (Paediatric focused triggering tool) eliminates the first 


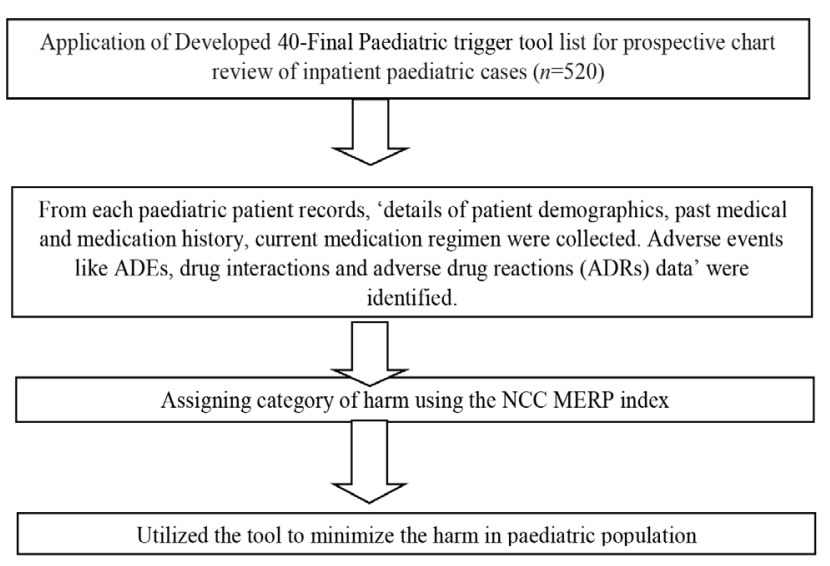

Figure 1: Prospective chart review of 520 paediatric case studies. $^{3}$

four categories of NCC MERP index as they consider the errors that do not cause harm. The present PFTT includes categories- E, F, G, H and I of the index as these categories consider the errors that cause harm.

\section{RESULTS}

Application of 40-Final Paediatric trigger tool with 5 modules developed using IHI-GTT to monthly random samples of 520 hospitalized paediatric patient was prospectively reviewed in the present study. Initial trigger tool with 61 triggers tools has been modified to final trigger tool of 40 Figure 1. Demographic characteristics of prospective chart review of patients $(N=520)$ was carried out with respect to frequency of Adverse Events (AE's) and harm, where 292 children were male and 228 were female. The incidence of adverse events was $35 \%$ and more among category of $1-5$ years of age (Table 1 and Graph 1 respectively). Total number of AE's and their incidence with respect to 5 modules were 448 triggers and mean rate of triggers per patient was 0.86 . AEs per 100 patient-days were $35 \%$ and 61.65 AEs per 1000 patient-days and two of the modules, care $(42.86 \%)$ and medication (36.81\%) had the maximum of harm events (Table 2). Out of 182 AEs identified, $107(58.80 \%)$ were found to be of E-category, $43(23.62 \%)$ were category F, 22(12.08\%) with category G, 9(4.95\%) with category $\mathrm{H}$ and $1(0.55 \%)$ was category I. (Table 3 and Graph 2). Two third of the paediatric population of category $\mathrm{E}$ and $\mathrm{F}(82.4 \%)$ had temporary harm (resulted in the intervention involving the treatment or initial/ prolonged hospitalization and has caused temporary harm) and remaining (17.6\%) were of non-preventable harm, category G-I (An error occurred that lead to the permanent patient harm, injury to the body organs or death). Minimizing the harm have also been highlighted in the present study (Table 4).

\begin{tabular}{|c|c|c|c|c|}
\hline \multicolumn{4}{|c|}{ Table 1: Demographic details. } \\
\hline \multirow{2}{*}{ Age(Days/year) } & \multicolumn{2}{|c|}{ Gender(N=520) } & \multicolumn{2}{|c|}{$\begin{array}{c}\text { Incidence } \\
\text { of Adverse } \\
\text { events(AE's), } \\
\boldsymbol{n}=\mathbf{1 8 2}\end{array}$} \\
\cline { 2 - 5 } & Male & Female & No.s & $\%$ \\
\hline $\mathbf{0 - 2 8}$ days & 65 & 54 & 28 & 15 \\
\hline $\mathbf{2 9 - 3 6 5}$ days & 74 & 49 & 42 & 24 \\
\hline $\mathbf{3 6 6}$ days to 5 years & 67 & 58 & 54 & 30 \\
\hline $\mathbf{5 - 1 2}$ years & 49 & 38 & 35 & 19 \\
\hline $\mathbf{1 2 - 1 8}$ years & 37 & 29 & 23 & 12 \\
\hline Total & 292 & 228 & 182 & $100 \%$ \\
\hline
\end{tabular}

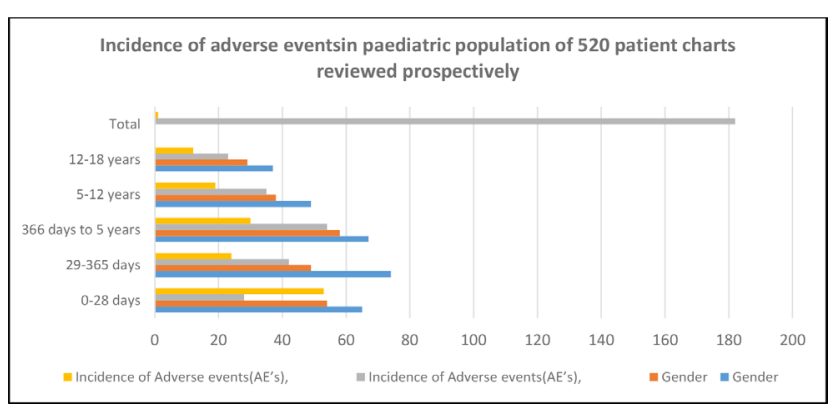

Graph 1: Incidence of adverse events in paediatric population of 520 patient charts reviewed prospectively.

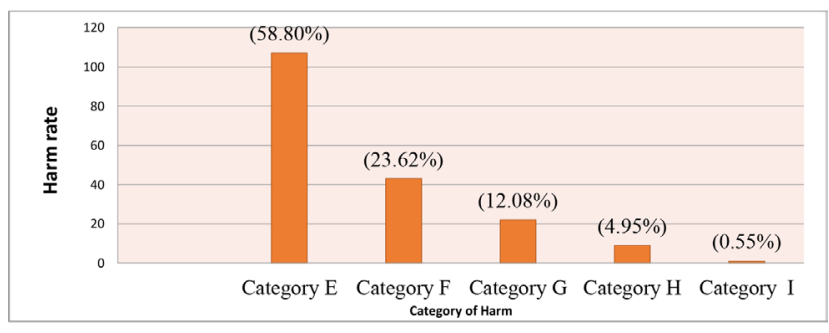

Graph 2: NCC MERP Harm Review (in \%).

\section{DISCUSSION}

Using the validated Paediatric focused trigger tool (PFTT) in the present study, incidence, types and severity of harm among children admitted to academic paediatric department of a tertiary care hospital was assessed. Incidence of 182 of harm, with 65 harm events per 1000 patient days and 35 harm events per 100 patient charts were in comparison with the study conducted by Kirkendall et al. where harm rate was 36.7 harm events per 100 patients and 76.3 per 1000 patient-days. ${ }^{3}$ Also, harm rate of 40 harm events per 100 patients and 54.9 per 1000 patient-days were identified in the study conducted by stockwell et al. ${ }^{5}$ Some other studies with higher harm rate within the paediatric intensive care population were of $26.1 \%$ to $62 \%{ }^{1,2,19,20}$ The results projected in the present and other studies 


\section{Table 2: Triggers with respect to no adverse events with severity of harm.}

\begin{tabular}{|c|c|c|c|c|c|c|c|c|c|c|}
\hline SI.no & Modules & Trigger names & $\begin{array}{l}\text { No. of } \\
\text { AE's }\end{array}$ & $\begin{array}{l}\text { No .of } \\
\text { Triggers }\end{array}$ & $\begin{array}{l}\text { AE \% } \\
\text { of PPV }\end{array}$ & \multicolumn{5}{|c|}{$\begin{array}{l}\text { Severity of harm as per } \\
\text { NCCMERP[E-I] }\end{array}$} \\
\hline \multicolumn{6}{|c|}{ 1. Care Module } & $\mathrm{E}$ & $\mathrm{F}$ & G & $\mathrm{H}$ & I \\
\hline 1 & PC1 & Transfusion/ use of blood products & 08 & 16 & 50 & 05 & 02 & 01 & 0 & 0 \\
\hline 2 & PC2 & Code/arrest/rapid response team & 02 & 09 & 33 & 0 & 00 & 01 & 01 & 0 \\
\hline 3 & PC3 & Positive blood culture & 05 & 11 & 45 & 04 & 01 & 0 & 0 & 0 \\
\hline 4 & PC4 & $\begin{array}{l}\text { x-ray or Doppler studies for emboli or } \\
\text { deep vein thrombosis }\end{array}$ & 04 & 10 & 36 & 04 & 0 & 0 & 0 & 0 \\
\hline 5 & PC5 & $\begin{array}{l}\text { Sudden Decrease in } 25 \% \text { in } \\
\text { haemoglobin or hematocrit value }\end{array}$ & 08 & 16 & 50 & 05 & 01 & 02 & 0 & 0 \\
\hline 6 & PC6 & Patient fall & 03 & 10 & 30 & 02 & 01 & 0 & 0 & 0 \\
\hline 7 & PC7 & Readmission within 30 days & 11 & 19 & 59 & 06 & 02 & 02 & 01 & 0 \\
\hline 8 & PC8 & $\begin{array}{l}\text { Health care associated infection of any } \\
\text { kind }\end{array}$ & 06 & 10 & 60 & 02 & 01 & 01 & 02 & 0 \\
\hline 9 & PC9 & Transfer to a higher level of care & 04 & 10 & 40 & 02 & 01 & 01 & 0 & 0 \\
\hline 10 & PC10 & Any procedure complication & 02 & 06 & 33 & 02 & 0 & 0 & 0 & 0 \\
\hline 11 & PC11 & Pain & 05 & 11 & 45 & 05 & 0 & 0 & 0 & 0 \\
\hline 12 & PC12 & Apgar score $<7$ & 04 & 09 & 44 & 02 & 01 & 01 & 0 & 0 \\
\hline 13 & PC13 & Abnormal body temperature & 06 & 13 & 46 & 04 & 02 & 0 & 0 & 0 \\
\hline 14 & PC14 & Loss of weight & 03 & 10 & 30 & 03 & 00 & 0 & 0 & 0 \\
\hline 15 & PC15 & Urinary retention & 03 & 10 & 30 & 02 & 01 & 0 & 0 & 0 \\
\hline 16 & PC16 & Fluid overload (pulmonary oedema) & 04 & 09 & 44 & 03 & 0 & 0 & 01 & 0 \\
\hline \multicolumn{3}{|c|}{ Total of all Care module: 16} & 78 & 177 & 44 & 51 & 13 & 09 & 05 & 0 \\
\hline \multicolumn{11}{|c|}{ 2. Medication (M) } \\
\hline 17 & PM1 & INR $>6 / \mathrm{PTT}>100 \mathrm{~s}$ & 01 & 03 & 33 & 00 & 01 & 0 & 0 & 0 \\
\hline 18 & PM2 & Glucose $<50 \mathrm{mg} / \mathrm{dL}$ & 04 & 08 & 50 & 02 & 02 & 0 & 0 & 0 \\
\hline 19 & PM3 & $\begin{array}{l}\text { Rise in BUN/ serum creatinine }>2 \text { times } \\
\text { of the normal. }\end{array}$ & 07 & 12 & 58 & 02 & 02 & 02 & 01 & 0 \\
\hline 20 & PM4 & Vitamin $\mathrm{K}$ administration & 02 & 14 & 14 & 01 & 01 & 0 & 0 & 0 \\
\hline 21 & PM5 & Diphenhydramine use & 11 & 22 & 50 & 08 & 02 & 01 & 0 & 0 \\
\hline 22 & PM6 & Antiemetic use & 06 & 12 & 50 & 04 & 02 & 0 & 0 & 0 \\
\hline 23 & PM7 & Over sedation/ hypotension & 02 & 08 & 25 & 01 & 01 & 0 & 0 & 0 \\
\hline 24 & PM8 & Abrupt medication stop & 11 & 21 & 52 & 09 & 01 & 01 & 0 & 0 \\
\hline 25 & PM9 & Use of stool softener / constipation & 12 & 26 & 46 & 07 & 02 & 02 & 01 & 0 \\
\hline 26 & PM10 & $\begin{array}{l}\text { Increased Glucose or administration of } \\
300 \text { or } 500 \mathrm{mg} / \mathrm{ml} \mathrm{of} \mathrm{glucose}\end{array}$ & 08 & 17 & 33 & 04 & 02 & 01 & 01 & 0 \\
\hline 27 & PM11 & Antidote administration & 03 & 07 & 42 & 02 & 01 & 0 & 0 & 0 \\
\hline \multicolumn{3}{|c|}{ Total of all medication module: 11} & 67 & 148 & 45 & 40 & 17 & 07 & 03 & 0 \\
\hline \multicolumn{11}{|c|}{ 3. Surgical (S) module } \\
\hline 28 & PS1 & $\begin{array}{l}\text { Intubation/re-intubation/bi level positive } \\
\text { airway pressure in PACU }\end{array}$ & 03 & 08 & 27 & 01 & 00 & 02 & 0 & 0 \\
\hline 29 & PS2 & $\begin{array}{l}\text { Mechanical ventilation }>24 \mathrm{~h} \\
\text { postoperatively }\end{array}$ & 02 & 08 & 33 & 0 & 01 & 01 & 0 & 0 \\
\hline 30 & PS3 & $\begin{array}{l}\text { Intraoperative epinephrine/ } \\
\text { norepinephrine. }\end{array}$ & 02 & 03 & 14 & 02 & 00 & 0 & 0 & 0 \\
\hline 31 & PS4 & Any operative complication & 01 & 06 & 33 & 0 & 0 & 0 & 0 & 01 \\
\hline \multicolumn{3}{|c|}{ Total of all Surgical module:04 } & 07 & 25 & 20 & 03 & 01 & 02 & 0 & 0 \\
\hline \multicolumn{11}{|c|}{ 4. Emergency Module(Perinatal+ intensive are merged) } \\
\hline 32 & PE1 & Readmission to ED within $48 \mathrm{~h}$ & 01 & 06 & 30 & 0 & 01 & 0 & 0 & 0 \\
\hline
\end{tabular}

\begin{tabular}{|c|c|c|c|c|c|c|c|c|c|c|}
\hline SI.no & Modules & Trigger names & $\begin{array}{l}\text { No. of } \\
\text { AE's }\end{array}$ & $\begin{array}{l}\text { No .of } \\
\text { Triggers }\end{array}$ & $\begin{array}{l}\text { AE \% } \\
\text { of PPV }\end{array}$ & \multicolumn{5}{|c|}{$\begin{array}{l}\text { Severity of harm as per } \\
\text { NCCMERP[E-I] }\end{array}$} \\
\hline \multicolumn{6}{|c|}{ 1. Care Module } & $\mathrm{E}$ & $\mathrm{F}$ & G & $\mathrm{H}$ & I \\
\hline 1 & PC1 & Transfusion/ use of blood products & 08 & 16 & 50 & 05 & 02 & 01 & 0 & 0 \\
\hline 2 & PC2 & Code/arrest/rapid response team & 02 & 09 & 33 & 0 & 00 & 01 & 01 & 0 \\
\hline 3 & PC3 & Positive blood culture & 05 & 11 & 45 & 04 & 01 & 0 & 0 & 0 \\
\hline 4 & PC4 & $\begin{array}{l}\text { x-ray or Doppler studies for emboli or } \\
\text { deep vein thrombosis }\end{array}$ & 04 & 10 & 36 & 04 & 0 & 0 & 0 & 0 \\
\hline 5 & PC5 & $\begin{array}{l}\text { Sudden Decrease in } 25 \% \text { in } \\
\text { haemoglobin or hematocrit value }\end{array}$ & 08 & 16 & 50 & 05 & 01 & 02 & 0 & 0 \\
\hline 6 & PC6 & Patient fall & 03 & 10 & 30 & 02 & 01 & 0 & 0 & 0 \\
\hline 7 & PC7 & Readmission within 30 days & 11 & 19 & 59 & 06 & 02 & 02 & 01 & 0 \\
\hline 8 & PC8 & $\begin{array}{l}\text { Health care associated infection of any } \\
\text { kind }\end{array}$ & 06 & 10 & 60 & 02 & 01 & 01 & 02 & 0 \\
\hline 9 & PC9 & Transfer to a higher level of care & 04 & 10 & 40 & 02 & 01 & 01 & 0 & 0 \\
\hline 10 & PC10 & Any procedure complication & 02 & 06 & 33 & 02 & 0 & 0 & 0 & 0 \\
\hline 11 & PC11 & Pain & 05 & 11 & 45 & 05 & 0 & 0 & 0 & 0 \\
\hline 12 & PC12 & Apgar score $<7$ & 04 & 09 & 44 & 02 & 01 & 01 & 0 & 0 \\
\hline 13 & PC13 & Abnormal body temperature & 06 & 13 & 46 & 04 & 02 & 0 & 0 & 0 \\
\hline 14 & PC14 & Loss of weight & 03 & 10 & 30 & 03 & 00 & 0 & 0 & 0 \\
\hline 15 & PC15 & Urinary retention & 03 & 10 & 30 & 02 & 01 & 0 & 0 & 0 \\
\hline 16 & PC16 & Fluid overload (pulmonary oedema) & 04 & 09 & 44 & 03 & 0 & 0 & 01 & 0 \\
\hline \multicolumn{3}{|c|}{ Total of all Care module: 16} & 78 & 177 & 44 & 51 & 13 & 09 & 05 & 0 \\
\hline \multicolumn{11}{|c|}{ 2. Medication (M) } \\
\hline 17 & PM1 & INR $>6 / \mathrm{PTT}>100 \mathrm{~s}$ & 01 & 03 & 33 & 00 & 01 & 0 & 0 & 0 \\
\hline 18 & PM2 & Glucose $<50 \mathrm{mg} / \mathrm{dL}$ & 04 & 08 & 50 & 02 & 02 & 0 & 0 & 0 \\
\hline 19 & PM3 & $\begin{array}{l}\text { Rise in BUN/ serum creatinine }>2 \text { times } \\
\text { of the normal. }\end{array}$ & 07 & 12 & 58 & 02 & 02 & 02 & 01 & 0 \\
\hline 20 & PM4 & Vitamin $\mathrm{K}$ administration & 02 & 14 & 14 & 01 & 01 & 0 & 0 & 0 \\
\hline 21 & PM5 & Diphenhydramine use & 11 & 22 & 50 & 08 & 02 & 01 & 0 & 0 \\
\hline 22 & PM6 & Antiemetic use & 06 & 12 & 50 & 04 & 02 & 0 & 0 & 0 \\
\hline 23 & PM7 & Over sedation/ hypotension & 02 & 08 & 25 & 01 & 01 & 0 & 0 & 0 \\
\hline 24 & PM8 & Abrupt medication stop & 11 & 21 & 52 & 09 & 01 & 01 & 0 & 0 \\
\hline 25 & PM9 & Use of stool softener / constipation & 12 & 26 & 46 & 07 & 02 & 02 & 01 & 0 \\
\hline 26 & PM10 & $\begin{array}{l}\text { Increased Glucose or administration of } \\
300 \text { or } 500 \mathrm{mg} / \mathrm{ml} \mathrm{of} \mathrm{glucose}\end{array}$ & 08 & 17 & 33 & 04 & 02 & 01 & 01 & 0 \\
\hline 27 & PM11 & Antidote administration & 03 & 07 & 42 & 02 & 01 & 0 & 0 & 0 \\
\hline \multicolumn{3}{|c|}{ Total of all medication module: 11} & 67 & 148 & 45 & 40 & 17 & 07 & 03 & 0 \\
\hline \multicolumn{11}{|c|}{ 3. Surgical (S) module } \\
\hline 28 & PS1 & $\begin{array}{l}\text { Intubation/re-intubation/bi level positive } \\
\text { airway pressure in PACU }\end{array}$ & 03 & 08 & 27 & 01 & 00 & 02 & 0 & 0 \\
\hline 29 & PS2 & $\begin{array}{l}\text { Mechanical ventilation }>24 \mathrm{~h} \\
\text { postoperatively }\end{array}$ & 02 & 08 & 33 & 0 & 01 & 01 & 0 & 0 \\
\hline 30 & PS3 & $\begin{array}{l}\text { Intraoperative epinephrine/ } \\
\text { norepinephrine. }\end{array}$ & 02 & 03 & 14 & 02 & 00 & 0 & 0 & 0 \\
\hline 31 & PS4 & Any operative complication & 01 & 06 & 33 & 0 & 0 & 0 & 0 & 01 \\
\hline \multicolumn{3}{|c|}{ Total of all Surgical module:04 } & 07 & 25 & 20 & 03 & 01 & 02 & 0 & 0 \\
\hline \multicolumn{11}{|c|}{ 4. Emergency Module(Perinatal+ intensive are merged) } \\
\hline 32 & PE1 & Readmission to ED within $48 \mathrm{~h}$ & 01 & 06 & 30 & 0 & 01 & 0 & 0 & 0 \\
\hline
\end{tabular}

\begin{tabular}{|c|c|c|c|c|c|c|c|c|c|c|}
\hline SI.no & Modules & Trigger names & $\begin{array}{l}\text { No. of } \\
\text { AE's }\end{array}$ & $\begin{array}{l}\text { No .of } \\
\text { Triggers }\end{array}$ & $\begin{array}{l}\text { AE \% } \\
\text { of PPV }\end{array}$ & \multicolumn{5}{|c|}{$\begin{array}{l}\text { Severity of harm as per } \\
\text { NCCMERP[E-I] }\end{array}$} \\
\hline \multicolumn{6}{|c|}{ 1. Care Module } & $\mathrm{E}$ & $\mathrm{F}$ & G & $\mathrm{H}$ & I \\
\hline 1 & PC1 & Transfusion/ use of blood products & 08 & 16 & 50 & 05 & 02 & 01 & 0 & 0 \\
\hline 2 & PC2 & Code/arrest/rapid response team & 02 & 09 & 33 & 0 & 00 & 01 & 01 & 0 \\
\hline 3 & PC3 & Positive blood culture & 05 & 11 & 45 & 04 & 01 & 0 & 0 & 0 \\
\hline 4 & PC4 & $\begin{array}{l}\text { x-ray or Doppler studies for emboli or } \\
\text { deep vein thrombosis }\end{array}$ & 04 & 10 & 36 & 04 & 0 & 0 & 0 & 0 \\
\hline 5 & PC5 & $\begin{array}{l}\text { Sudden Decrease in } 25 \% \text { in } \\
\text { haemoglobin or hematocrit value }\end{array}$ & 08 & 16 & 50 & 05 & 01 & 02 & 0 & 0 \\
\hline 6 & PC6 & Patient fall & 03 & 10 & 30 & 02 & 01 & 0 & 0 & 0 \\
\hline 7 & PC7 & Readmission within 30 days & 11 & 19 & 59 & 06 & 02 & 02 & 01 & 0 \\
\hline 8 & PC8 & $\begin{array}{l}\text { Health care associated infection of any } \\
\text { kind }\end{array}$ & 06 & 10 & 60 & 02 & 01 & 01 & 02 & 0 \\
\hline 9 & PC9 & Transfer to a higher level of care & 04 & 10 & 40 & 02 & 01 & 01 & 0 & 0 \\
\hline 10 & PC10 & Any procedure complication & 02 & 06 & 33 & 02 & 0 & 0 & 0 & 0 \\
\hline 11 & PC11 & Pain & 05 & 11 & 45 & 05 & 0 & 0 & 0 & 0 \\
\hline 12 & PC12 & Apgar score $<7$ & 04 & 09 & 44 & 02 & 01 & 01 & 0 & 0 \\
\hline 13 & PC13 & Abnormal body temperature & 06 & 13 & 46 & 04 & 02 & 0 & 0 & 0 \\
\hline 14 & PC14 & Loss of weight & 03 & 10 & 30 & 03 & 00 & 0 & 0 & 0 \\
\hline 15 & PC15 & Urinary retention & 03 & 10 & 30 & 02 & 01 & 0 & 0 & 0 \\
\hline 16 & PC16 & Fluid overload (pulmonary oedema) & 04 & 09 & 44 & 03 & 0 & 0 & 01 & 0 \\
\hline \multicolumn{3}{|c|}{ Total of all Care module: 16} & 78 & 177 & 44 & 51 & 13 & 09 & 05 & 0 \\
\hline \multicolumn{11}{|c|}{ 2. Medication (M) } \\
\hline 17 & PM1 & INR $>6 / \mathrm{PTT}>100 \mathrm{~s}$ & 01 & 03 & 33 & 00 & 01 & 0 & 0 & 0 \\
\hline 18 & PM2 & Glucose $<50 \mathrm{mg} / \mathrm{dL}$ & 04 & 08 & 50 & 02 & 02 & 0 & 0 & 0 \\
\hline 19 & PM3 & $\begin{array}{l}\text { Rise in BUN/ serum creatinine }>2 \text { times } \\
\text { of the normal. }\end{array}$ & 07 & 12 & 58 & 02 & 02 & 02 & 01 & 0 \\
\hline 20 & PM4 & Vitamin $\mathrm{K}$ administration & 02 & 14 & 14 & 01 & 01 & 0 & 0 & 0 \\
\hline 21 & PM5 & Diphenhydramine use & 11 & 22 & 50 & 08 & 02 & 01 & 0 & 0 \\
\hline 22 & PM6 & Antiemetic use & 06 & 12 & 50 & 04 & 02 & 0 & 0 & 0 \\
\hline 23 & PM7 & Over sedation/ hypotension & 02 & 08 & 25 & 01 & 01 & 0 & 0 & 0 \\
\hline 24 & PM8 & Abrupt medication stop & 11 & 21 & 52 & 09 & 01 & 01 & 0 & 0 \\
\hline 25 & PM9 & Use of stool softener / constipation & 12 & 26 & 46 & 07 & 02 & 02 & 01 & 0 \\
\hline 26 & PM10 & $\begin{array}{l}\text { Increased Glucose or administration of } \\
300 \text { or } 500 \mathrm{mg} / \mathrm{ml} \mathrm{of} \mathrm{glucose}\end{array}$ & 08 & 17 & 33 & 04 & 02 & 01 & 01 & 0 \\
\hline 27 & PM11 & Antidote administration & 03 & 07 & 42 & 02 & 01 & 0 & 0 & 0 \\
\hline \multicolumn{3}{|c|}{ Total of all medication module: 11} & 67 & 148 & 45 & 40 & 17 & 07 & 03 & 0 \\
\hline \multicolumn{11}{|c|}{ 3. Surgical (S) module } \\
\hline 28 & PS1 & $\begin{array}{l}\text { Intubation/re-intubation/bi level positive } \\
\text { airway pressure in PACU }\end{array}$ & 03 & 08 & 27 & 01 & 00 & 02 & 0 & 0 \\
\hline 29 & PS2 & $\begin{array}{l}\text { Mechanical ventilation }>24 \mathrm{~h} \\
\text { postoperatively }\end{array}$ & 02 & 08 & 33 & 0 & 01 & 01 & 0 & 0 \\
\hline 30 & PS3 & $\begin{array}{l}\text { Intraoperative epinephrine/ } \\
\text { norepinephrine. }\end{array}$ & 02 & 03 & 14 & 02 & 00 & 0 & 0 & 0 \\
\hline 31 & PS4 & Any operative complication & 01 & 06 & 33 & 0 & 0 & 0 & 0 & 01 \\
\hline \multicolumn{3}{|c|}{ Total of all Surgical module:04 } & 07 & 25 & 20 & 03 & 01 & 02 & 0 & 0 \\
\hline \multicolumn{11}{|c|}{ 4. Emergency Module(Perinatal+ intensive are merged) } \\
\hline 32 & PE1 & Readmission to ED within $48 \mathrm{~h}$ & 01 & 06 & 30 & 0 & 01 & 0 & 0 & 0 \\
\hline
\end{tabular}

\begin{tabular}{|c|c|c|c|c|c|c|c|c|c|c|}
\hline SI.no & Modules & Trigger names & $\begin{array}{l}\text { No. of } \\
\text { AE's }\end{array}$ & $\begin{array}{l}\text { No .of } \\
\text { Triggers }\end{array}$ & $\begin{array}{l}\text { AE \% } \\
\text { of PPV }\end{array}$ & \multicolumn{5}{|c|}{$\begin{array}{l}\text { Severity of harm as per } \\
\text { NCCMERP[E-I] }\end{array}$} \\
\hline \multicolumn{6}{|c|}{ 1. Care Module } & $\mathrm{E}$ & $\mathrm{F}$ & G & $\mathrm{H}$ & I \\
\hline 1 & PC1 & Transfusion/ use of blood products & 08 & 16 & 50 & 05 & 02 & 01 & 0 & 0 \\
\hline 2 & $\mathrm{PC2}$ & Code/arrest/rapid response team & 02 & 09 & 33 & 0 & 00 & 01 & 01 & 0 \\
\hline 3 & PC3 & Positive blood culture & 05 & 11 & 45 & 04 & 01 & 0 & 0 & 0 \\
\hline 4 & PC4 & $\begin{array}{c}\text { x-ray or Doppler studies for emboli or } \\
\text { deep vein thrombosis }\end{array}$ & 04 & 10 & 36 & 04 & 0 & 0 & 0 & 0 \\
\hline 5 & PC5 & $\begin{array}{l}\text { Sudden Decrease in } 25 \% \text { in } \\
\text { haemoglobin or hematocrit value }\end{array}$ & 08 & 16 & 50 & 05 & 01 & 02 & 0 & 0 \\
\hline 6 & PC6 & Patient fall & 03 & 10 & 30 & 02 & 01 & 0 & 0 & 0 \\
\hline 7 & PC7 & Readmission within 30 days & 11 & 19 & 59 & 06 & 02 & 02 & 01 & 0 \\
\hline 8 & PC8 & $\begin{array}{l}\text { Health care associated infection of any } \\
\text { kind }\end{array}$ & 06 & 10 & 60 & 02 & 01 & 01 & 02 & 0 \\
\hline 9 & PC9 & Transfer to a higher level of care & 04 & 10 & 40 & 02 & 01 & 01 & 0 & 0 \\
\hline 10 & PC10 & Any procedure complication & 02 & 06 & 33 & 02 & 0 & 0 & 0 & 0 \\
\hline 11 & PC11 & Pain & 05 & 11 & 45 & 05 & 0 & 0 & 0 & 0 \\
\hline 12 & PC12 & Apgar score $<7$ & 04 & 09 & 44 & 02 & 01 & 01 & 0 & 0 \\
\hline 13 & PC13 & Abnormal body temperature & 06 & 13 & 46 & 04 & 02 & 0 & 0 & 0 \\
\hline 14 & PC14 & Loss of weight & 03 & 10 & 30 & 03 & 00 & 0 & 0 & 0 \\
\hline 15 & PC15 & Urinary retention & 03 & 10 & 30 & 02 & 01 & 0 & 0 & 0 \\
\hline 16 & PC16 & Fluid overload (pulmonary oedema) & 04 & 09 & 44 & 03 & 0 & 0 & 01 & 0 \\
\hline \multicolumn{3}{|c|}{ Total of all Care module: 16} & 78 & 177 & 44 & 51 & 13 & 09 & 05 & 0 \\
\hline \multicolumn{11}{|c|}{ 2. Medication (M) } \\
\hline 17 & PM1 & INR $>6 / \mathrm{PTT}>100 \mathrm{~s}$ & 01 & 03 & 33 & 00 & 01 & 0 & 0 & 0 \\
\hline 18 & PM2 & Glucose $<50 \mathrm{mg} / \mathrm{dL}$ & 04 & 08 & 50 & 02 & 02 & 0 & 0 & 0 \\
\hline 19 & PM3 & $\begin{array}{c}\text { Rise in BUN/ serum creatinine }>2 \text { times } \\
\text { of the normal. }\end{array}$ & 07 & 12 & 58 & 02 & 02 & 02 & 01 & 0 \\
\hline 20 & PM4 & Vitamin $\mathrm{K}$ administration & 02 & 14 & 14 & 01 & 01 & 0 & 0 & 0 \\
\hline 21 & PM5 & Diphenhydramine use & 11 & 22 & 50 & 08 & 02 & 01 & 0 & 0 \\
\hline 22 & PM6 & Antiemetic use & 06 & 12 & 50 & 04 & 02 & 0 & 0 & 0 \\
\hline 23 & PM7 & Over sedation/ hypotension & 02 & 08 & 25 & 01 & 01 & 0 & 0 & 0 \\
\hline 24 & PM8 & Abrupt medication stop & 11 & 21 & 52 & 09 & 01 & 01 & 0 & 0 \\
\hline 25 & PM9 & Use of stool softener / constipation & 12 & 26 & 46 & 07 & 02 & 02 & 01 & 0 \\
\hline 26 & PM10 & $\begin{array}{l}\text { Increased Glucose or administration of } \\
300 \text { or } 500 \mathrm{mg} / \mathrm{ml} \text { of glucose }\end{array}$ & 08 & 17 & 33 & 04 & 02 & 01 & 01 & 0 \\
\hline 27 & PM11 & Antidote administration & 03 & 07 & 42 & 02 & 01 & 0 & 0 & 0 \\
\hline \multicolumn{3}{|c|}{ Total of all medication module: 11} & 67 & 148 & 45 & 40 & 17 & 07 & 03 & 0 \\
\hline \multicolumn{11}{|c|}{ 3. Surgical (S) module } \\
\hline 28 & PS1 & $\begin{array}{c}\text { Intubation/re-intubation/bi level positive } \\
\text { airway pressure in PACU }\end{array}$ & 03 & 08 & 27 & 01 & 00 & 02 & 0 & 0 \\
\hline 29 & PS2 & $\begin{array}{l}\text { Mechanical ventilation }>24 \mathrm{~h} \\
\text { postoperatively }\end{array}$ & 02 & 08 & 33 & 0 & 01 & 01 & 0 & 0 \\
\hline 30 & PS3 & $\begin{array}{l}\text { Intraoperative epinephrine/ } \\
\text { norepinephrine. }\end{array}$ & 02 & 03 & 14 & 02 & 00 & 0 & 0 & 0 \\
\hline 31 & PS4 & Any operative complication & 01 & 06 & 33 & 0 & 0 & 0 & 0 & 01 \\
\hline \multicolumn{3}{|c|}{ Total of all Surgical module:04 } & 07 & 25 & 20 & 03 & 01 & 02 & 0 & 0 \\
\hline \multicolumn{11}{|c|}{ 4. Emergency Module(Perinatal+ intensive are merged) } \\
\hline 32 & PE1 & Readmission to ED within $48 \mathrm{~h}$ & 01 & 06 & 30 & 0 & 01 & 0 & 0 & 0 \\
\hline
\end{tabular}

\begin{tabular}{|c|c|c|c|c|c|c|c|c|c|c|}
\hline SI.no & Modules & Trigger names & $\begin{array}{l}\text { No. of } \\
\text { AE's }\end{array}$ & $\begin{array}{l}\text { No .of } \\
\text { Triggers }\end{array}$ & $\begin{array}{l}\text { AE \% } \\
\text { of PPV }\end{array}$ & \multicolumn{5}{|c|}{$\begin{array}{l}\text { Severity of harm as per } \\
\text { NCCMERP[E-I] }\end{array}$} \\
\hline \multicolumn{6}{|c|}{ 1. Care Module } & $\mathrm{E}$ & $\mathrm{F}$ & G & $\mathrm{H}$ & I \\
\hline 1 & PC1 & Transfusion/ use of blood products & 08 & 16 & 50 & 05 & 02 & 01 & 0 & 0 \\
\hline 2 & PC2 & Code/arrest/rapid response team & 02 & 09 & 33 & 0 & 00 & 01 & 01 & 0 \\
\hline 3 & PC3 & Positive blood culture & 05 & 11 & 45 & 04 & 01 & 0 & 0 & 0 \\
\hline 4 & PC4 & $\begin{array}{l}\text { x-ray or Doppler studies for emboli or } \\
\text { deep vein thrombosis }\end{array}$ & 04 & 10 & 36 & 04 & 0 & 0 & 0 & 0 \\
\hline 5 & PC5 & $\begin{array}{l}\text { Sudden Decrease in } 25 \% \text { in } \\
\text { haemoglobin or hematocrit value }\end{array}$ & 08 & 16 & 50 & 05 & 01 & 02 & 0 & 0 \\
\hline 6 & PC6 & Patient fall & 03 & 10 & 30 & 02 & 01 & 0 & 0 & 0 \\
\hline 7 & PC7 & Readmission within 30 days & 11 & 19 & 59 & 06 & 02 & 02 & 01 & 0 \\
\hline 8 & PC8 & $\begin{array}{l}\text { Health care associated infection of any } \\
\text { kind }\end{array}$ & 06 & 10 & 60 & 02 & 01 & 01 & 02 & 0 \\
\hline 9 & PC9 & Transfer to a higher level of care & 04 & 10 & 40 & 02 & 01 & 01 & 0 & 0 \\
\hline 10 & PC10 & Any procedure complication & 02 & 06 & 33 & 02 & 0 & 0 & 0 & 0 \\
\hline 11 & PC11 & Pain & 05 & 11 & 45 & 05 & 0 & 0 & 0 & 0 \\
\hline 12 & PC12 & Apgar score $<7$ & 04 & 09 & 44 & 02 & 01 & 01 & 0 & 0 \\
\hline 13 & PC13 & Abnormal body temperature & 06 & 13 & 46 & 04 & 02 & 0 & 0 & 0 \\
\hline 14 & PC14 & Loss of weight & 03 & 10 & 30 & 03 & 00 & 0 & 0 & 0 \\
\hline 15 & PC15 & Urinary retention & 03 & 10 & 30 & 02 & 01 & 0 & 0 & 0 \\
\hline 16 & PC16 & Fluid overload (pulmonary oedema) & 04 & 09 & 44 & 03 & 0 & 0 & 01 & 0 \\
\hline \multicolumn{3}{|c|}{ Total of all Care module: 16} & 78 & 177 & 44 & 51 & 13 & 09 & 05 & 0 \\
\hline \multicolumn{11}{|c|}{ 2. Medication (M) } \\
\hline 17 & PM1 & INR $>6 / \mathrm{PTT}>100 \mathrm{~s}$ & 01 & 03 & 33 & 00 & 01 & 0 & 0 & 0 \\
\hline 18 & PM2 & Glucose $<50 \mathrm{mg} / \mathrm{dL}$ & 04 & 08 & 50 & 02 & 02 & 0 & 0 & 0 \\
\hline 19 & PM3 & $\begin{array}{l}\text { Rise in BUN/ serum creatinine }>2 \text { times } \\
\text { of the normal. }\end{array}$ & 07 & 12 & 58 & 02 & 02 & 02 & 01 & 0 \\
\hline 20 & PM4 & Vitamin $\mathrm{K}$ administration & 02 & 14 & 14 & 01 & 01 & 0 & 0 & 0 \\
\hline 21 & PM5 & Diphenhydramine use & 11 & 22 & 50 & 08 & 02 & 01 & 0 & 0 \\
\hline 22 & PM6 & Antiemetic use & 06 & 12 & 50 & 04 & 02 & 0 & 0 & 0 \\
\hline 23 & PM7 & Over sedation/ hypotension & 02 & 08 & 25 & 01 & 01 & 0 & 0 & 0 \\
\hline 24 & PM8 & Abrupt medication stop & 11 & 21 & 52 & 09 & 01 & 01 & 0 & 0 \\
\hline 25 & PM9 & Use of stool softener / constipation & 12 & 26 & 46 & 07 & 02 & 02 & 01 & 0 \\
\hline 26 & PM10 & $\begin{array}{l}\text { Increased Glucose or administration of } \\
300 \text { or } 500 \mathrm{mg} / \mathrm{ml} \text { of glucose }\end{array}$ & 08 & 17 & 33 & 04 & 02 & 01 & 01 & 0 \\
\hline 27 & PM11 & Antidote administration & 03 & 07 & 42 & 02 & 01 & 0 & 0 & 0 \\
\hline \multicolumn{3}{|c|}{ Total of all medication module: 11} & 67 & 148 & 45 & 40 & 17 & 07 & 03 & 0 \\
\hline \multicolumn{11}{|c|}{ 3. Surgical (S) module } \\
\hline 28 & PS1 & $\begin{array}{l}\text { Intubation/re-intubation/bi level positive } \\
\text { airway pressure in PACU }\end{array}$ & 03 & 08 & 27 & 01 & 00 & 02 & 0 & 0 \\
\hline 29 & PS2 & $\begin{array}{l}\text { Mechanical ventilation }>24 \mathrm{~h} \\
\text { postoperatively }\end{array}$ & 02 & 08 & 33 & 0 & 01 & 01 & 0 & 0 \\
\hline 30 & PS3 & $\begin{array}{l}\text { Intraoperative epinephrine/ } \\
\text { norepinephrine. }\end{array}$ & 02 & 03 & 14 & 02 & 00 & 0 & 0 & 0 \\
\hline 31 & PS4 & Any operative complication & 01 & 06 & 33 & 0 & 0 & 0 & 0 & 01 \\
\hline \multicolumn{3}{|c|}{ Total of all Surgical module:04 } & 07 & 25 & 20 & 03 & 01 & 02 & 0 & 0 \\
\hline \multicolumn{11}{|c|}{ 4. Emergency Module(Perinatal+ intensive are merged) } \\
\hline 32 & PE1 & Readmission to ED within $48 \mathrm{~h}$ & 01 & 06 & 30 & 0 & 01 & 0 & 0 & 0 \\
\hline
\end{tabular}

\begin{tabular}{|c|c|c|c|c|c|c|c|c|c|c|}
\hline SI.no & Modules & Trigger names & $\begin{array}{l}\text { No. of } \\
\text { AE's }\end{array}$ & $\begin{array}{l}\text { No .of } \\
\text { Triggers }\end{array}$ & $\begin{array}{l}\text { AE \% } \\
\text { of PPV }\end{array}$ & \multicolumn{5}{|c|}{$\begin{array}{l}\text { Severity of harm as per } \\
\text { NCCMERP[E-I] }\end{array}$} \\
\hline \multicolumn{6}{|c|}{ 1. Care Module } & $\mathrm{E}$ & $\mathrm{F}$ & G & $\mathrm{H}$ & I \\
\hline 1 & PC1 & Transfusion/ use of blood products & 08 & 16 & 50 & 05 & 02 & 01 & 0 & 0 \\
\hline 2 & PC2 & Code/arrest/rapid response team & 02 & 09 & 33 & 0 & 00 & 01 & 01 & 0 \\
\hline 3 & PC3 & Positive blood culture & 05 & 11 & 45 & 04 & 01 & 0 & 0 & 0 \\
\hline 4 & PC4 & $\begin{array}{l}\text { x-ray or Doppler studies for emboli or } \\
\text { deep vein thrombosis }\end{array}$ & 04 & 10 & 36 & 04 & 0 & 0 & 0 & 0 \\
\hline 5 & PC5 & $\begin{array}{l}\text { Sudden Decrease in } 25 \% \text { in } \\
\text { haemoglobin or hematocrit value }\end{array}$ & 08 & 16 & 50 & 05 & 01 & 02 & 0 & 0 \\
\hline 6 & PC6 & Patient fall & 03 & 10 & 30 & 02 & 01 & 0 & 0 & 0 \\
\hline 7 & PC7 & Readmission within 30 days & 11 & 19 & 59 & 06 & 02 & 02 & 01 & 0 \\
\hline 8 & PC8 & $\begin{array}{l}\text { Health care associated infection of any } \\
\text { kind }\end{array}$ & 06 & 10 & 60 & 02 & 01 & 01 & 02 & 0 \\
\hline 9 & PC9 & Transfer to a higher level of care & 04 & 10 & 40 & 02 & 01 & 01 & 0 & 0 \\
\hline 10 & PC10 & Any procedure complication & 02 & 06 & 33 & 02 & 0 & 0 & 0 & 0 \\
\hline 11 & PC11 & Pain & 05 & 11 & 45 & 05 & 0 & 0 & 0 & 0 \\
\hline 12 & PC12 & Apgar score $<7$ & 04 & 09 & 44 & 02 & 01 & 01 & 0 & 0 \\
\hline 13 & PC13 & Abnormal body temperature & 06 & 13 & 46 & 04 & 02 & 0 & 0 & 0 \\
\hline 14 & PC14 & Loss of weight & 03 & 10 & 30 & 03 & 00 & 0 & 0 & 0 \\
\hline 15 & PC15 & Urinary retention & 03 & 10 & 30 & 02 & 01 & 0 & 0 & 0 \\
\hline 16 & PC16 & Fluid overload (pulmonary oedema) & 04 & 09 & 44 & 03 & 0 & 0 & 01 & 0 \\
\hline \multicolumn{3}{|c|}{ Total of all Care module: 16} & 78 & 177 & 44 & 51 & 13 & 09 & 05 & 0 \\
\hline \multicolumn{11}{|c|}{ 2. Medication (M) } \\
\hline 17 & PM1 & INR $>6 / \mathrm{PTT}>100 \mathrm{~s}$ & 01 & 03 & 33 & 00 & 01 & 0 & 0 & 0 \\
\hline 18 & PM2 & Glucose $<50 \mathrm{mg} / \mathrm{dL}$ & 04 & 08 & 50 & 02 & 02 & 0 & 0 & 0 \\
\hline 19 & PM3 & $\begin{array}{l}\text { Rise in BUN/ serum creatinine }>2 \text { times } \\
\text { of the normal. }\end{array}$ & 07 & 12 & 58 & 02 & 02 & 02 & 01 & 0 \\
\hline 20 & PM4 & Vitamin $\mathrm{K}$ administration & 02 & 14 & 14 & 01 & 01 & 0 & 0 & 0 \\
\hline 21 & PM5 & Diphenhydramine use & 11 & 22 & 50 & 08 & 02 & 01 & 0 & 0 \\
\hline 22 & PM6 & Antiemetic use & 06 & 12 & 50 & 04 & 02 & 0 & 0 & 0 \\
\hline 23 & PM7 & Over sedation/ hypotension & 02 & 08 & 25 & 01 & 01 & 0 & 0 & 0 \\
\hline 24 & PM8 & Abrupt medication stop & 11 & 21 & 52 & 09 & 01 & 01 & 0 & 0 \\
\hline 25 & PM9 & Use of stool softener / constipation & 12 & 26 & 46 & 07 & 02 & 02 & 01 & 0 \\
\hline 26 & PM10 & $\begin{array}{l}\text { Increased Glucose or administration of } \\
300 \text { or } 500 \mathrm{mg} / \mathrm{ml} \mathrm{of} \mathrm{glucose}\end{array}$ & 08 & 17 & 33 & 04 & 02 & 01 & 01 & 0 \\
\hline 27 & PM11 & Antidote administration & 03 & 07 & 42 & 02 & 01 & 0 & 0 & 0 \\
\hline \multicolumn{3}{|c|}{ Total of all medication module:11 } & 67 & 148 & 45 & 40 & 17 & 07 & 03 & 0 \\
\hline \multicolumn{11}{|c|}{ 3. Surgical (S) module } \\
\hline 28 & PS1 & $\begin{array}{l}\text { Intubation/re-intubation/bi level positive } \\
\text { airway pressure in PACU }\end{array}$ & 03 & 08 & 27 & 01 & 00 & 02 & 0 & 0 \\
\hline 29 & PS2 & $\begin{array}{l}\text { Mechanical ventilation }>24 \mathrm{~h} \\
\text { postoperatively }\end{array}$ & 02 & 08 & 33 & 0 & 01 & 01 & 0 & 0 \\
\hline 30 & PS3 & $\begin{array}{l}\text { Intraoperative epinephrine/ } \\
\text { norepinephrine. }\end{array}$ & 02 & 03 & 14 & 02 & 00 & 0 & 0 & 0 \\
\hline 31 & PS4 & Any operative complication & 01 & 06 & 33 & 0 & 0 & 0 & 0 & 01 \\
\hline \multicolumn{3}{|c|}{ Total of all Surgical module:04 } & 07 & 25 & 20 & 03 & 01 & 02 & 0 & 0 \\
\hline \multicolumn{11}{|c|}{ 4. Emergency Module(Perinatal+ intensive are merged) } \\
\hline 32 & PE1 & Readmission to ED within $48 \mathrm{~h}$ & 01 & 06 & 30 & 0 & 01 & 0 & 0 & 0 \\
\hline
\end{tabular}

\begin{tabular}{|c|c|c|c|c|c|c|c|c|c|c|}
\hline SI.no & Modules & Trigger names & $\begin{array}{l}\text { No. of } \\
\text { AE's }\end{array}$ & $\begin{array}{l}\text { No .of } \\
\text { Triggers }\end{array}$ & $\begin{array}{l}\text { AE \% } \\
\text { of PPV }\end{array}$ & \multicolumn{5}{|c|}{$\begin{array}{l}\text { Severity of harm as per } \\
\text { NCCMERP[E-I] }\end{array}$} \\
\hline \multicolumn{6}{|c|}{ 1. Care Module } & $\mathrm{E}$ & $\mathrm{F}$ & G & $\mathrm{H}$ & I \\
\hline 1 & PC1 & Transfusion/ use of blood products & 08 & 16 & 50 & 05 & 02 & 01 & 0 & 0 \\
\hline 2 & PC2 & Code/arrest/rapid response team & 02 & 09 & 33 & 0 & 00 & 01 & 01 & 0 \\
\hline 3 & PC3 & Positive blood culture & 05 & 11 & 45 & 04 & 01 & 0 & 0 & 0 \\
\hline 4 & PC4 & $\begin{array}{l}\text { x-ray or Doppler studies for emboli or } \\
\text { deep vein thrombosis }\end{array}$ & 04 & 10 & 36 & 04 & 0 & 0 & 0 & 0 \\
\hline 5 & PC5 & $\begin{array}{l}\text { Sudden Decrease in } 25 \% \text { in } \\
\text { haemoglobin or hematocrit value }\end{array}$ & 08 & 16 & 50 & 05 & 01 & 02 & 0 & 0 \\
\hline 6 & PC6 & Patient fall & 03 & 10 & 30 & 02 & 01 & 0 & 0 & 0 \\
\hline 7 & PC7 & Readmission within 30 days & 11 & 19 & 59 & 06 & 02 & 02 & 01 & 0 \\
\hline 8 & PC8 & $\begin{array}{l}\text { Health care associated infection of any } \\
\text { kind }\end{array}$ & 06 & 10 & 60 & 02 & 01 & 01 & 02 & 0 \\
\hline 9 & PC9 & Transfer to a higher level of care & 04 & 10 & 40 & 02 & 01 & 01 & 0 & 0 \\
\hline 10 & PC10 & Any procedure complication & 02 & 06 & 33 & 02 & 0 & 0 & 0 & 0 \\
\hline 11 & PC11 & Pain & 05 & 11 & 45 & 05 & 0 & 0 & 0 & 0 \\
\hline 12 & PC12 & Apgar score $<7$ & 04 & 09 & 44 & 02 & 01 & 01 & 0 & 0 \\
\hline 13 & PC13 & Abnormal body temperature & 06 & 13 & 46 & 04 & 02 & 0 & 0 & 0 \\
\hline 14 & PC14 & Loss of weight & 03 & 10 & 30 & 03 & 00 & 0 & 0 & 0 \\
\hline 15 & PC15 & Urinary retention & 03 & 10 & 30 & 02 & 01 & 0 & 0 & 0 \\
\hline 16 & PC16 & Fluid overload (pulmonary oedema) & 04 & 09 & 44 & 03 & 0 & 0 & 01 & 0 \\
\hline \multicolumn{3}{|c|}{ Total of all Care module: 16} & 78 & 177 & 44 & 51 & 13 & 09 & 05 & 0 \\
\hline \multicolumn{11}{|c|}{ 2. Medication (M) } \\
\hline 17 & PM1 & INR $>6 / \mathrm{PTT}>100 \mathrm{~s}$ & 01 & 03 & 33 & 00 & 01 & 0 & 0 & 0 \\
\hline 18 & PM2 & Glucose $<50 \mathrm{mg} / \mathrm{dL}$ & 04 & 08 & 50 & 02 & 02 & 0 & 0 & 0 \\
\hline 19 & PM3 & $\begin{array}{l}\text { Rise in BUN/ serum creatinine }>2 \text { times } \\
\text { of the normal. }\end{array}$ & 07 & 12 & 58 & 02 & 02 & 02 & 01 & 0 \\
\hline 20 & PM4 & Vitamin $\mathrm{K}$ administration & 02 & 14 & 14 & 01 & 01 & 0 & 0 & 0 \\
\hline 21 & PM5 & Diphenhydramine use & 11 & 22 & 50 & 08 & 02 & 01 & 0 & 0 \\
\hline 22 & PM6 & Antiemetic use & 06 & 12 & 50 & 04 & 02 & 0 & 0 & 0 \\
\hline 23 & PM7 & Over sedation/ hypotension & 02 & 08 & 25 & 01 & 01 & 0 & 0 & 0 \\
\hline 24 & PM8 & Abrupt medication stop & 11 & 21 & 52 & 09 & 01 & 01 & 0 & 0 \\
\hline 25 & PM9 & Use of stool softener / constipation & 12 & 26 & 46 & 07 & 02 & 02 & 01 & 0 \\
\hline 26 & PM10 & $\begin{array}{l}\text { Increased Glucose or administration of } \\
300 \text { or } 500 \mathrm{mg} / \mathrm{ml} \mathrm{of} \mathrm{glucose}\end{array}$ & 08 & 17 & 33 & 04 & 02 & 01 & 01 & 0 \\
\hline 27 & PM11 & Antidote administration & 03 & 07 & 42 & 02 & 01 & 0 & 0 & 0 \\
\hline \multicolumn{3}{|c|}{ Total of all medication module: 11} & 67 & 148 & 45 & 40 & 17 & 07 & 03 & 0 \\
\hline \multicolumn{11}{|c|}{ 3. Surgical (S) module } \\
\hline 28 & PS1 & $\begin{array}{l}\text { Intubation/re-intubation/bi level positive } \\
\text { airway pressure in PACU }\end{array}$ & 03 & 08 & 27 & 01 & 00 & 02 & 0 & 0 \\
\hline 29 & PS2 & $\begin{array}{l}\text { Mechanical ventilation }>24 \mathrm{~h} \\
\text { postoperatively }\end{array}$ & 02 & 08 & 33 & 0 & 01 & 01 & 0 & 0 \\
\hline 30 & PS3 & $\begin{array}{l}\text { Intraoperative epinephrine/ } \\
\text { norepinephrine. }\end{array}$ & 02 & 03 & 14 & 02 & 00 & 0 & 0 & 0 \\
\hline 31 & PS4 & Any operative complication & 01 & 06 & 33 & 0 & 0 & 0 & 0 & 01 \\
\hline \multicolumn{3}{|c|}{ Total of all Surgical module:04 } & 07 & 25 & 20 & 03 & 01 & 02 & 0 & 0 \\
\hline \multicolumn{11}{|c|}{ 4. Emergency Module(Perinatal+ intensive are merged) } \\
\hline 32 & PE1 & Readmission to ED within $48 \mathrm{~h}$ & 01 & 06 & 30 & 0 & 01 & 0 & 0 & 0 \\
\hline
\end{tabular}

\begin{tabular}{|c|c|c|c|c|c|c|c|c|c|c|}
\hline SI.no & Modules & Trigger names & $\begin{array}{l}\text { No. of } \\
\text { AE's }\end{array}$ & $\begin{array}{l}\text { No .of } \\
\text { Triggers }\end{array}$ & $\begin{array}{l}\text { AE \% } \\
\text { of PPV }\end{array}$ & \multicolumn{5}{|c|}{$\begin{array}{l}\text { Severity of harm as per } \\
\text { NCCMERP[E-I] }\end{array}$} \\
\hline \multicolumn{6}{|c|}{ 1. Care Module } & $\mathrm{E}$ & $\mathrm{F}$ & G & $\mathrm{H}$ & I \\
\hline 1 & PC1 & Transfusion/ use of blood products & 08 & 16 & 50 & 05 & 02 & 01 & 0 & 0 \\
\hline 2 & PC2 & Code/arrest/rapid response team & 02 & 09 & 33 & 0 & 00 & 01 & 01 & 0 \\
\hline 3 & PC3 & Positive blood culture & 05 & 11 & 45 & 04 & 01 & 0 & 0 & 0 \\
\hline 4 & PC4 & $\begin{array}{l}\text { x-ray or Doppler studies for emboli or } \\
\text { deep vein thrombosis }\end{array}$ & 04 & 10 & 36 & 04 & 0 & 0 & 0 & 0 \\
\hline 5 & PC5 & $\begin{array}{l}\text { Sudden Decrease in } 25 \% \text { in } \\
\text { haemoglobin or hematocrit value }\end{array}$ & 08 & 16 & 50 & 05 & 01 & 02 & 0 & 0 \\
\hline 6 & PC6 & Patient fall & 03 & 10 & 30 & 02 & 01 & 0 & 0 & 0 \\
\hline 7 & PC7 & Readmission within 30 days & 11 & 19 & 59 & 06 & 02 & 02 & 01 & 0 \\
\hline 8 & PC8 & $\begin{array}{l}\text { Health care associated infection of any } \\
\text { kind }\end{array}$ & 06 & 10 & 60 & 02 & 01 & 01 & 02 & 0 \\
\hline 9 & PC9 & Transfer to a higher level of care & 04 & 10 & 40 & 02 & 01 & 01 & 0 & 0 \\
\hline 10 & PC10 & Any procedure complication & 02 & 06 & 33 & 02 & 0 & 0 & 0 & 0 \\
\hline 11 & PC11 & Pain & 05 & 11 & 45 & 05 & 0 & 0 & 0 & 0 \\
\hline 12 & PC12 & Apgar score $<7$ & 04 & 09 & 44 & 02 & 01 & 01 & 0 & 0 \\
\hline 13 & PC13 & Abnormal body temperature & 06 & 13 & 46 & 04 & 02 & 0 & 0 & 0 \\
\hline 14 & PC14 & Loss of weight & 03 & 10 & 30 & 03 & 00 & 0 & 0 & 0 \\
\hline 15 & PC15 & Urinary retention & 03 & 10 & 30 & 02 & 01 & 0 & 0 & 0 \\
\hline 16 & PC16 & Fluid overload (pulmonary oedema) & 04 & 09 & 44 & 03 & 0 & 0 & 01 & 0 \\
\hline \multicolumn{3}{|c|}{ Total of all Care module: 16} & 78 & 177 & 44 & 51 & 13 & 09 & 05 & 0 \\
\hline \multicolumn{11}{|c|}{ 2. Medication (M) } \\
\hline 17 & PM1 & INR $>6 / \mathrm{PTT}>100 \mathrm{~s}$ & 01 & 03 & 33 & 00 & 01 & 0 & 0 & 0 \\
\hline 18 & PM2 & Glucose $<50 \mathrm{mg} / \mathrm{dL}$ & 04 & 08 & 50 & 02 & 02 & 0 & 0 & 0 \\
\hline 19 & PM3 & $\begin{array}{l}\text { Rise in BUN/ serum creatinine }>2 \text { times } \\
\text { of the normal. }\end{array}$ & 07 & 12 & 58 & 02 & 02 & 02 & 01 & 0 \\
\hline 20 & PM4 & Vitamin $\mathrm{K}$ administration & 02 & 14 & 14 & 01 & 01 & 0 & 0 & 0 \\
\hline 21 & PM5 & Diphenhydramine use & 11 & 22 & 50 & 08 & 02 & 01 & 0 & 0 \\
\hline 22 & PM6 & Antiemetic use & 06 & 12 & 50 & 04 & 02 & 0 & 0 & 0 \\
\hline 23 & PM7 & Over sedation/ hypotension & 02 & 08 & 25 & 01 & 01 & 0 & 0 & 0 \\
\hline 24 & PM8 & Abrupt medication stop & 11 & 21 & 52 & 09 & 01 & 01 & 0 & 0 \\
\hline 25 & PM9 & Use of stool softener / constipation & 12 & 26 & 46 & 07 & 02 & 02 & 01 & 0 \\
\hline 26 & PM10 & $\begin{array}{l}\text { Increased Glucose or administration of } \\
300 \text { or } 500 \mathrm{mg} / \mathrm{ml} \mathrm{of} \mathrm{glucose}\end{array}$ & 08 & 17 & 33 & 04 & 02 & 01 & 01 & 0 \\
\hline 27 & PM11 & Antidote administration & 03 & 07 & 42 & 02 & 01 & 0 & 0 & 0 \\
\hline \multicolumn{3}{|c|}{ Total of all medication module: 11} & 67 & 148 & 45 & 40 & 17 & 07 & 03 & 0 \\
\hline \multicolumn{11}{|c|}{ 3. Surgical (S) module } \\
\hline 28 & PS1 & $\begin{array}{l}\text { Intubation/re-intubation/bi level positive } \\
\text { airway pressure in PACU }\end{array}$ & 03 & 08 & 27 & 01 & 00 & 02 & 0 & 0 \\
\hline 29 & PS2 & $\begin{array}{l}\text { Mechanical ventilation }>24 \mathrm{~h} \\
\text { postoperatively }\end{array}$ & 02 & 08 & 33 & 0 & 01 & 01 & 0 & 0 \\
\hline 30 & PS3 & $\begin{array}{l}\text { Intraoperative epinephrine/ } \\
\text { norepinephrine. }\end{array}$ & 02 & 03 & 14 & 02 & 00 & 0 & 0 & 0 \\
\hline 31 & PS4 & Any operative complication & 01 & 06 & 33 & 0 & 0 & 0 & 0 & 01 \\
\hline \multicolumn{3}{|c|}{ Total of all Surgical module:04 } & 07 & 25 & 20 & 03 & 01 & 02 & 0 & 0 \\
\hline \multicolumn{11}{|c|}{ 4. Emergency Module(Perinatal+ intensive are merged) } \\
\hline 32 & PE1 & Readmission to ED within $48 \mathrm{~h}$ & 01 & 06 & 30 & 0 & 01 & 0 & 0 & 0 \\
\hline
\end{tabular}

\begin{tabular}{|c|c|c|c|c|c|c|c|c|c|c|}
\hline SI.no & Modules & Trigger names & $\begin{array}{l}\text { No. of } \\
\text { AE's }\end{array}$ & $\begin{array}{l}\text { No .of } \\
\text { Triggers }\end{array}$ & $\begin{array}{l}\text { AE \% } \\
\text { of PPV }\end{array}$ & \multicolumn{5}{|c|}{$\begin{array}{l}\text { Severity of harm as per } \\
\text { NCCMERP[E-I] }\end{array}$} \\
\hline \multicolumn{6}{|c|}{ 1. Care Module } & $\mathrm{E}$ & $\mathrm{F}$ & G & $\mathrm{H}$ & I \\
\hline 1 & PC1 & Transfusion/ use of blood products & 08 & 16 & 50 & 05 & 02 & 01 & 0 & 0 \\
\hline 2 & PC2 & Code/arrest/rapid response team & 02 & 09 & 33 & 0 & 00 & 01 & 01 & 0 \\
\hline 3 & PC3 & Positive blood culture & 05 & 11 & 45 & 04 & 01 & 0 & 0 & 0 \\
\hline 4 & PC4 & $\begin{array}{l}\text { x-ray or Doppler studies for emboli or } \\
\text { deep vein thrombosis }\end{array}$ & 04 & 10 & 36 & 04 & 0 & 0 & 0 & 0 \\
\hline 5 & PC5 & $\begin{array}{l}\text { Sudden Decrease in } 25 \% \text { in } \\
\text { haemoglobin or hematocrit value }\end{array}$ & 08 & 16 & 50 & 05 & 01 & 02 & 0 & 0 \\
\hline 6 & PC6 & Patient fall & 03 & 10 & 30 & 02 & 01 & 0 & 0 & 0 \\
\hline 7 & PC7 & Readmission within 30 days & 11 & 19 & 59 & 06 & 02 & 02 & 01 & 0 \\
\hline 8 & PC8 & $\begin{array}{l}\text { Health care associated infection of any } \\
\text { kind }\end{array}$ & 06 & 10 & 60 & 02 & 01 & 01 & 02 & 0 \\
\hline 9 & PC9 & Transfer to a higher level of care & 04 & 10 & 40 & 02 & 01 & 01 & 0 & 0 \\
\hline 10 & PC10 & Any procedure complication & 02 & 06 & 33 & 02 & 0 & 0 & 0 & 0 \\
\hline 11 & PC11 & Pain & 05 & 11 & 45 & 05 & 0 & 0 & 0 & 0 \\
\hline 12 & PC12 & Apgar score $<7$ & 04 & 09 & 44 & 02 & 01 & 01 & 0 & 0 \\
\hline 13 & PC13 & Abnormal body temperature & 06 & 13 & 46 & 04 & 02 & 0 & 0 & 0 \\
\hline 14 & PC14 & Loss of weight & 03 & 10 & 30 & 03 & 00 & 0 & 0 & 0 \\
\hline 15 & PC15 & Urinary retention & 03 & 10 & 30 & 02 & 01 & 0 & 0 & 0 \\
\hline 16 & PC16 & Fluid overload (pulmonary oedema) & 04 & 09 & 44 & 03 & 0 & 0 & 01 & 0 \\
\hline \multicolumn{3}{|c|}{ Total of all Care module: 16} & 78 & 177 & 44 & 51 & 13 & 09 & 05 & 0 \\
\hline \multicolumn{11}{|c|}{ 2. Medication (M) } \\
\hline 17 & PM1 & INR $>6 / \mathrm{PTT}>100 \mathrm{~s}$ & 01 & 03 & 33 & 00 & 01 & 0 & 0 & 0 \\
\hline 18 & PM2 & Glucose $<50 \mathrm{mg} / \mathrm{dL}$ & 04 & 08 & 50 & 02 & 02 & 0 & 0 & 0 \\
\hline 19 & PM3 & $\begin{array}{l}\text { Rise in BUN/ serum creatinine }>2 \text { times } \\
\text { of the normal. }\end{array}$ & 07 & 12 & 58 & 02 & 02 & 02 & 01 & 0 \\
\hline 20 & PM4 & Vitamin $\mathrm{K}$ administration & 02 & 14 & 14 & 01 & 01 & 0 & 0 & 0 \\
\hline 21 & PM5 & Diphenhydramine use & 11 & 22 & 50 & 08 & 02 & 01 & 0 & 0 \\
\hline 22 & PM6 & Antiemetic use & 06 & 12 & 50 & 04 & 02 & 0 & 0 & 0 \\
\hline 23 & PM7 & Over sedation/ hypotension & 02 & 08 & 25 & 01 & 01 & 0 & 0 & 0 \\
\hline 24 & PM8 & Abrupt medication stop & 11 & 21 & 52 & 09 & 01 & 01 & 0 & 0 \\
\hline 25 & PM9 & Use of stool softener / constipation & 12 & 26 & 46 & 07 & 02 & 02 & 01 & 0 \\
\hline 26 & PM10 & $\begin{array}{l}\text { Increased Glucose or administration of } \\
300 \text { or } 500 \mathrm{mg} / \mathrm{ml} \mathrm{of} \mathrm{glucose}\end{array}$ & 08 & 17 & 33 & 04 & 02 & 01 & 01 & 0 \\
\hline 27 & PM11 & Antidote administration & 03 & 07 & 42 & 02 & 01 & 0 & 0 & 0 \\
\hline \multicolumn{3}{|c|}{ Total of all medication module: 11} & 67 & 148 & 45 & 40 & 17 & 07 & 03 & 0 \\
\hline \multicolumn{11}{|c|}{ 3. Surgical (S) module } \\
\hline 28 & PS1 & $\begin{array}{l}\text { Intubation/re-intubation/bi level positive } \\
\text { airway pressure in PACU }\end{array}$ & 03 & 08 & 27 & 01 & 00 & 02 & 0 & 0 \\
\hline 29 & PS2 & $\begin{array}{l}\text { Mechanical ventilation }>24 \mathrm{~h} \\
\text { postoperatively }\end{array}$ & 02 & 08 & 33 & 0 & 01 & 01 & 0 & 0 \\
\hline 30 & PS3 & $\begin{array}{l}\text { Intraoperative epinephrine/ } \\
\text { norepinephrine. }\end{array}$ & 02 & 03 & 14 & 02 & 00 & 0 & 0 & 0 \\
\hline 31 & PS4 & Any operative complication & 01 & 06 & 33 & 0 & 0 & 0 & 0 & 01 \\
\hline \multicolumn{3}{|c|}{ Total of all Surgical module:04 } & 07 & 25 & 20 & 03 & 01 & 02 & 0 & 0 \\
\hline \multicolumn{11}{|c|}{ 4. Emergency Module(Perinatal+ intensive are merged) } \\
\hline 32 & PE1 & Readmission to ED within $48 \mathrm{~h}$ & 01 & 06 & 30 & 0 & 01 & 0 & 0 & 0 \\
\hline
\end{tabular}




\begin{tabular}{|c|c|c|c|c|c|c|c|c|c|c|}
\hline \multirow{2}{*}{\begin{tabular}{|c|} 
SI.no \\
33 \\
\end{tabular}} & \multirow{2}{*}{$\begin{array}{l}\text { Modules } \\
\text { PE2 }\end{array}$} & \multirow{2}{*}{$\begin{array}{c}\text { Trigger names } \\
\text { Pneumonia onset }\end{array}$} & \multirow{2}{*}{$\begin{array}{c}\begin{array}{c}\text { No. of } \\
\text { AE's }\end{array} \\
03 \\
\end{array}$} & \multirow{2}{*}{\begin{tabular}{|c|}
$\begin{array}{c}\text { No .of } \\
\text { Triggers }\end{array}$ \\
06
\end{tabular}} & \multirow{2}{*}{$\begin{array}{c}\text { AE \% } \\
\text { of PPV } \\
\text { triggers }\end{array}$} & \multicolumn{5}{|c|}{$\begin{array}{l}\text { Severity of harm as per } \\
\text { NCCMERP[E-I] }\end{array}$} \\
\hline & & & & & & 01 & 01 & 01 & 0 & 0 \\
\hline \multicolumn{3}{|c|}{ Total of all Emergency Module:02 } & 04 & 12 & 23 & 01 & 02 & 01 & 0 & 0 \\
\hline \multicolumn{11}{|c|}{ 5. Laboratory module } \\
\hline 34 & PL1 & Platelet count $<50,000$ & 04 & 15 & 27 & 02 & 01 & 00 & 01 & 0 \\
\hline 35 & PL2 & Extreme hyper bilirubinaemia & 06 & 16 & 38 & 02 & 03 & 01 & 0 & 0 \\
\hline 36 & PL3 & Neutropenia and antibiotic treatment & 03 & 09 & 33 & 0 & 02 & 01 & 0 & 0 \\
\hline 37 & PL4 & Abnormal liver enzymes & 05 & 11 & 45 & 04 & 01 & 00 & 0 & 0 \\
\hline 38 & PL5 & Sodium: $120 \mathrm{mmol} / \mathrm{l}>\mathrm{Na}>150 \mathrm{mmol} / \mathrm{l}$ & 03 & 09 & 33 & 01 & 01 & 01 & 0 & 0 \\
\hline 39 & PL6 & Potassium: $3.0 \mathrm{mmol} / \mathrm{l}>\mathrm{K}>6.0 \mathrm{mmol} / \mathrm{l}$ & 03 & 07 & 42 & 02 & 01 & 00 & 0 & 0 \\
\hline 40 & PL7 & $\begin{array}{c}\text { Leucopenia } \\
\text { Age }<1 \text { month WBC }<5000 / \mathrm{mm}^{3}\left(5 \times 10^{9} / \mathrm{l}\right) \\
\text { Age } 1-23 \text { months } \mathrm{WBC}<4000 / \mathrm{mm}^{3} \\
\left(4 \times 10^{9} / \mathrm{l}\right) \\
\text { Age } 2-18 \text { years } \mathrm{WBC}<3000 / \mathrm{mm}^{3} \\
\left(3.0310^{9} / \mathrm{l}\right)\end{array}$ & 02 & 06 & 33 & 01 & 01 & 0 & 0 & 0 \\
\hline \multicolumn{3}{|c|}{ Total of all laboratory Module:07 } & 26 & 73 & 36 & 12 & 10 & 03 & 01 & 01 \\
\hline \multicolumn{3}{|c|}{ Overall total } & 182 & 448 & 41 & 107 & 43 & 22 & 09 & 01 \\
\hline
\end{tabular}

\begin{tabular}{|c|c|c|}
\hline $\begin{array}{l}\text { NCC MERP } \\
\text { Scale }\end{array}$ & Definition of category of harm & $\begin{array}{l}\text { NCC MERP } \\
\text { harm rate }\end{array}$ \\
\hline Category- E & An error occurred that resulted in the treatment or intervention and lead to temporary harm & 107 \\
\hline Category- F & An error occurred that resulted in the initial or prolonged hospitalization but lead to temporary harm & 43 \\
\hline Category- G & An error occurred that resulted in the permanent patient harm like injury to the organs & 22 \\
\hline Category- H & An error occurred that lead to a near death event (e.g severe cutaneous reactions, cardiac arrest) & 09 \\
\hline \multirow[t]{2}{*}{ Category - I } & An error occurred that lead to patient death & 01 \\
\hline & Total & 182 \\
\hline
\end{tabular}

\begin{tabular}{|c|c|c|c|c|}
\hline $\begin{array}{l}\text { SI. } \\
\text { No. }\end{array}$ & Adverse events & Harm & $\begin{array}{c}\text { Preventabilityl } \\
\text { Non- preventability (E-I) }\end{array}$ & Harm and its minimization \\
\hline 1. & $\begin{array}{l}\text { Insertion of nasogastric } \\
\text { feeding tube }\end{array}$ & $\begin{array}{l}\text { Lead to temporary } \\
\text { harm }\end{array}$ & E (Preventable) & $\begin{array}{l}\text { Required intervention and medication } \\
\text { management }\end{array}$ \\
\hline 2. & $\begin{array}{l}\text { Decrease in } 25 \% \text { of } \\
\text { Haemoglobin }\end{array}$ & $\begin{array}{l}\text { Lead to temporary } \\
\text { harm }\end{array}$ & E (Preventable) & $\begin{array}{l}\text { Required intervention and transfusion of } \\
\text { blood. }\end{array}$ \\
\hline 3. & $\begin{array}{l}\text { Transfer to an intensive } \\
\text { level of care }\end{array}$ & $\begin{array}{l}\text { Lead to permanent } \\
\text { harm }\end{array}$ & F (Non-Preventable) & $\begin{array}{l}\text { Emergency care and strict monitoring } \\
\text { was done }\end{array}$ \\
\hline 4. & Heparin use & Bleeding & E (Preventable) & $\begin{array}{c}\text { Vit. K was administered and INR } \\
\text { monitored }\end{array}$ \\
\hline 5. & Amoxycillin+ Clavulinic acid & Diarrhoea /Vomiting & E (Preventable) & Use of anti-diarrhoeal medication. \\
\hline 6. & Insulin dose & Hypoglycemia & E (Preventable) & $\begin{array}{l}\text { Dose titrated and Hypoglycemia was } \\
\text { addressed }\end{array}$ \\
\hline 7. & Sodium valproate & $\begin{array}{l}\text { Steven's Jhonson } \\
\text { syndrome }\end{array}$ & $\mathrm{H}$ (Non-preventable) & $\begin{array}{c}\text { Drug was stopped, Intensive supportive } \\
\text { care treatment was started }\end{array}$ \\
\hline 8. & Influenza Vaccine & Anaphylactic shock & $\mathrm{H}$ (Non-preventable) & $\begin{array}{c}\text { Intensive supportive care treatment was } \\
\text { started }\end{array}$ \\
\hline
\end{tabular}


reflected that, academic teaching hospitals or institutions probably represent higher rate of harm in paediatric patient due to diversity of population and disease severity. This was in contrast with the another study by Solevag et al. where, 21 harm events per 1000 patient days and 6 harm events per 100 patient consultations were found, the reason being 3 months short duration of study. ${ }^{21}$

Majority of the paediatric population of category E and $\mathrm{F}(82.4 \%)$ had temporary harm (required treatment or intervention and lead to temporary harm) whereas and remaining $(17.6 \%)$ had non-preventable harm (permanent harm) in the present study. This was in similar with other studies like Takata et al..$^{22}$ where, it was reported that $97 \%$ of AEs discovered were of category E, with the remainders in the category F. Kirkendall et al. study represents $76 \%$ of AEs of category E, 22\% were category $\mathrm{F}$ and $2 \%$ were category H. where majority $(92.3 \%)$ represented temporary harm while severe harm was rare of $7.8 \%$ of the harm identified. $^{2}$

\section{CONCLUSION}

The present study highlights on the rate of harm assessed significantly among paediatric population with the help of developed Paediatric-Focused Trigger Tool (PFTT). This tool was modified using adult Global trigger tool (GTT), where triggers with low predictive value were deleted and with high predictive value were retained/added, to develop focused final trigger tool which was used to assess the harm more accurately in a measurable level experienced by children admitted in the hospital. Range of triggers will help with paediatric set ups to assess their usefulness and develop strategies to mitigate them. This emphasizes on the monitoring process and inter disciplinary approach towards mitigating the harm.

\section{ACKNOWLEDGEMENT}

The authors thank the Department of Paediatrics and head of the Paediatrics department, KLEs Dr. Prabhakar Kore Hospital and Medical research centre for supporting in carrying out the research work and providing valuable feedback and also to Medical records department to collect health records of patients.

\section{CONFLICT OF INTEREST}

The authors declare no conflict of interest.

\section{ABBREVIATIONS}

AE: Adverse events; PPV: Positive predictive Value; NCCMERP: National Coordinating Council for Medication Error Reporting and Prevention; PC: Paediatric Care; PM: Paediatric Medication; PS: Paediatric Surgery; PE: Paediatric Emergency; PL: Paediatric Laboratory; APGAR: Appearance, Pulse, Grimace, Activity and Respiration; WBC: White blood cells; ED: Emergency Department; PTT: Partial Thromboplastin Time; BUN: Blood Urea Nitrogen;

PACU: Post Anaesthesia Care Unit.

\section{REFERENCES}

1. Matlow AG, Cronin CM, Flintoft V, Nijssen-Jordan C, Fleming M, et al. Description of the development and validation of the Canadian Paediatric Trigger Tool. BMJ Qual Saf. 2011;20(5):416-23.

2. Kirkendall ES, Kloppenborg E, Papp J, White D, Frese C, Hacker D, et al. Measuring adverse events and levels of harm in pediatric inpatients with the Global Trigger Tool. Pediatrics. 2012;130(5). [cited 2015 NOV 05]. Available from:http://www.pediatrics.org/cgi/ content/full/130/5/130/5/e1206.

3. Griffin FA, Resar RK. IHI Global Trigger Tool for Measuring Adverse Events. IHI Innovation Series White Paper. Cambridge, MA: Institute for Healthcare Improvement. 2009. [cited 2015 JAN 11]. Available from: http://www.IHI.org.

4. Stockwell DC, Bisarya $\mathrm{H}$, Classen DC, Kirkendall ES, Landrigan CP, Lemon V, et al. A Trigger Tool to Detect Harm in Pediatric Inpatient Settings. Pediatrics. 2015;135(6):1036-42.

5. Stockwell DC, Slonim AD. Quality and safety in the intensive care unit. J Intensive Care Med. 2006;21(4):199-210.

6. Classen DC, Pestotnik SL, Evans RS. Computerized surveillance of adverse drug events in hospital patients. JAMA. 1991;266(20):2847-51.

7. Jha AK, Kuperman GJ, Teich JM, Jha AK, Kuperman GJ, Teich JM, et al. Identifying adverse drug events: Development of a computer-based monitor and comparison with chart review and stimulated voluntary report. J Am Med Inform Assoc. 1998;5(3):305-14.

8. Olsen S, Neale G, Schwab K, Psaila B, Patel T, Chapman EJ, et al. Hospital staff should use more than one method to detect adverse events and potential adverse events: Incident reporting, pharmacist surveillance and local real-time record review may all have a place. Qual Saf Health Care. 2007;16(1):40-4.

9. Wetzels R, René W, Chris WV, Michel W. Mix of methods is needed to identify adverse events in general practice: A prospective observational study. BMC Fam Pract. 2008;9:35

10. Naessens JM, Campbell CR, Huddleston JM. A comparison of hospital adverse events identified by three widely used detection methods. Int J Qual Health Care. 2009;21(4):301-7.

11. Ferranti J, Horvath MM, Cozart H, Whitehurst J, Eckstrand J. Reevaluating the safety profile of paediatrics: A comparison of computerized adverse drug event surveillance and voluntary reporting in the paediatric environment. Paediatrics. 2008;121(5):1201-7.

12. Brennan TA, Leape LL, Laird NM, Hebert L, Localio AR, Lawthers AG, et al. Incidence of adverse events and negligence in hospitalized patients. Results of the Harvard Medical Practice Study I N Engl J M. 1991;324(6):370-6.

13. Resar RK, Rozich JD, Classen D. Methodology and rationale for the measurement of harm with trigger tools. Qual Saf Health Care. 2003;12(2):39-45.

14. Rozich JD, Haraden CR, Resar RK. Adverse drug event trigger tool: A practical methodology for measuring medication related harm. Qual Saf Health Care. 2003;12(3):194-200.

15. Jick H. Drugs-remarkably nontoxic. New England Journal of Medicine. 1974;291(16):824-8 
16. Peter ML, Leslie AM, Stephen PT, Karen JB, Evelyn MK, James AM, et al. Patient safety efforts should focus on medical injuries. Journal of the American Medical Association. 2002;287(15):1993-7.

17. WHO. World Health Organization. Patient safety, Strengthening capacity for patient safety research. 2014b. [cited 14 MAR 2017]Available from: http:// www.who.int/patient safety/research/strengthening capacity/measuring harm/ en/ health-topics/Health-systems/patient-safety.

18. National Coordinating Council for Medication Error Reporting and Prevention. Types of Medication Errors. [cited 24 JUNE 2017]. Available from: http:// www. nccmerp. Org /med Error CatIndex.html. Updated February 20, 2001.

19. Larsen GY, Donaldson AE, Parker HB. Preventable harm occurring to critically ill children. Pediatr Crit Care Med. 2007;8(4):331-6.
20. Agarwal S, Classen D, Larsen G, Tofil NM, Hayes LW, Sullivan JE, et al. Prevalence of adverse events in pediatric intensive care units in the United States. Pediatr Crit Care Med. 2010;11(5):568-78.

21. Solevåg AL, Nakstad B. Utility of a Paediatric Trigger Tool in a Norwegian department of Paediatric and adolescent medicine. BMJ Open. 2014;4(5):e005011.

22. Takata GS, Mason W, Taketomo C, Logsdon T, Sharek PJ. Development, testing and findings of a pediatric-focused trigger tool to identify medicationrelated harm in US children's hospitals. Pediatrics. 2008;121(4):e927-35. [cited 16 AUG 2016]. Available from: http:// www.pediatrics.org/cgi/ content/ full/121/4/e927-e937.

\section{PICTORIAL ABSTRACT}

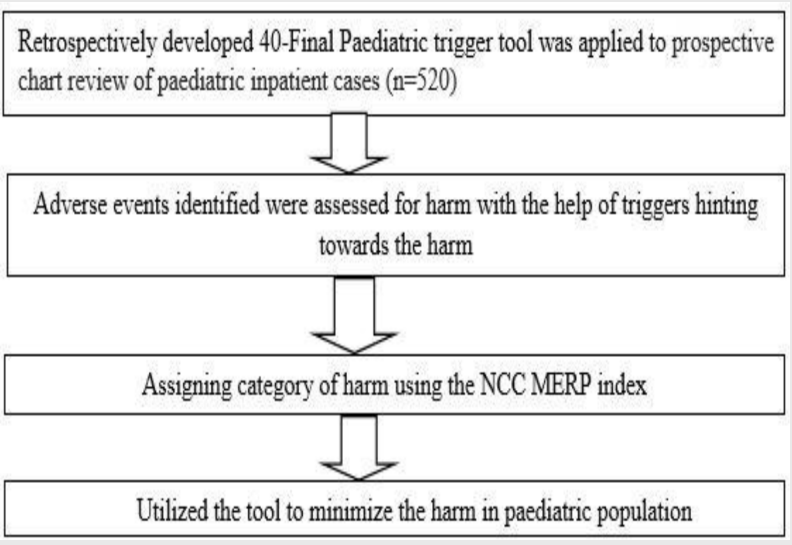

\section{SUMMARY}

- Patient safety among the children still remains to be a concern, especially in the developing countries as data necessary to asses harm is still lacking.

- Hence, it becomes a prerequisite to generate ideas and plans to improve the safety by developing strategies to assess and mitigate the harm in the paediatric population.

\section{About Authors}

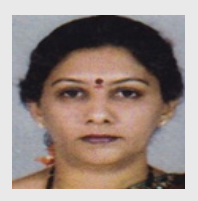

Mrs Geetanjali S Salimath Assistant Professor, Dept. of Pharmacy Practice KLE College of Pharmacy, KLE Academy for Higher Education and Research (KAHER), Belagavi, Karnataka, India-590010. Scientific member, Institutional Ethics Committee, KLE Academy for higher education and Research (KAHER) Belagavi-10 BOS member for M.Sc in clinical research.

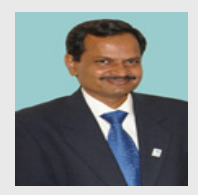

Prof. (Dr.) M.S Ganachari Head, Department of Pharmacy Practice, KLE College of Pharmacy, KLE Academy for Higher Education and Research (KAHER) Belagavi, Karnataka, India-590010. Also designated as a Deputy registrar, KLE Academy for Higher Education and Research (KAHER). Member Secretary, IEC, KAHER. Research Pharmacist, KLEU Research Unit in collaboration with $\mathrm{NIH}$ and Gates Foundation Board of studies member with various academic bodies with 26 years of teaching experience.

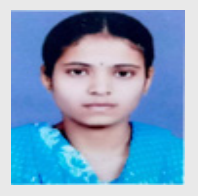

Ms. Manjula G, Assistant Professor Department of Pharmacy Practice KLE College of Pharmacy, KLE Academy for Higher Education and Research (KAHER) Belagavi, Karnataka, India-590010. Her area of interest is identifying and mitigating drug dosing problems in Paediatric oncology patients.

Cite this article: Salimath GS, Ganachari MS, Manjula G. Paediatric Focused Triggering Tool (PFTT) To Assess the Harm and its Utilization to Minimize the Levels of Harm among Children at a Tertiary Care Hospital. Indian J of Pharmaceutical Education and Research. 2020;54(3):819-25. 